\title{
Study of Simple but Challenging Diffusion Equation
}

\author{
Daniel W. Stroock
}

M.I.T., 2-272, Cambridge, MA 02139-4307, USA, dws@math.mit.edu, The author aknowledges support from NSF Grant DMS 0244991

Summary. This note contains a summary of results, obtained in collaboration with David Williams, about a simple diffusion equation which does not fit the standard probabilistic model. In particular, the usual minimum principle does not apply, and its absence gives rise to some phenomena which are, at least to us, both unfamiliar and interesting.

\section{Introduction}

For the past two years David Williams and I have been devoting an embarrassing amount of effort ${ }^{1}$ to understand solutions to the linear, constant coefficient partial differential equation

$$
\dot{u}=\frac{1}{2} u^{\prime \prime}+\mu u^{\prime} \text { in } I \times[0, \infty) \text { with } \dot{u}(t, 0)=\sigma u^{\prime}(t, 0) \text { for } t \in I,
$$

where $\dot{u}=\partial_{t} u$ and $u^{\prime}=\partial_{x} u, I \subseteq \mathbb{R}$ is an open interval, and $(\mu, \sigma) \in \mathbb{R}^{2}$. It is essential for our analysis that a solution $u$ be continuously differentiable at least once in $t$ and twice in $x$ in the whole of $I \times[0, \infty)$, including the spacial boundary $I \times\{0\}$.

Our initial result (cf. Theorem 1.1 in [?]) deals with the Cauchy initial value problem for (1). In its statement, the set $F$ of initial values consists of bounded $f:[0, \infty) \longrightarrow \mathbb{R}$ which are continuous on $(0, \infty)$, but not necessarily at 0 . The set $U$ from which solutions come consists of $u \in C^{1,2}((0, \infty) \times[0, \infty) ; \mathbb{R})$ which are bounded in $(0,1) \times[0, \infty)$ and have the property that $\dot{u}$ and $u^{\prime \prime}$ are bounded on each vertical slice $\left[T_{1}, T_{2}\right] \times[0, \infty)$, where $0<T_{1}<T_{2}<\infty$.

Theorem 1. Suppose that $u \in U$ satisfies (1) in $(0, \infty) \times[0, \infty)$ and that $f(x) \equiv \lim _{t \backslash 0} u(t, x)$ exists for each $x \in(0, \infty)$. Then $f(0)=\lim _{t \backslash 0} u(t, 0)$

\footnotetext{
${ }^{1}$ As explained in [?], our original reason for looking at these equations came from the study of certain Wiener-Hopf decompositions. However, our continued study of them has been motivated by pure intellectual curiosity.
} 
exists and the convergence of $u(t, \cdot)$ to $f$ takes place uniformly on compact subsets of $(0, \infty)$. In particular, $f \in F$. Conversely, for each $f \in F$ there is a unique solution $u=u_{f} \in U$ to (1) such that $\lim _{t \backslash 0} u(t, x)=f(x)$ for each $x \in[0, \infty)$, and the convergence is uniform on compact subsets of $(0, \infty)$. In particular, if $\mathbf{Q}_{t} f \equiv u_{f}(t, \cdot)$ for $t>0$ and $f \in F$, then, for each $t>0, \mathbf{Q}_{t}$ maps $F$

boundedly into $C_{b}([0, \infty) ; \mathbb{R})$ and $\left\{\mathbf{Q}_{t}: t \geq 0\right\}$ is a semigroup. Finally, if $\left\{f_{n}\right\}_{1}^{\infty} \subseteq F$ is a bounded sequence which tends to $f \in F$ in the sense that $f_{n}(0) \longrightarrow f(0)$ and $f_{n} \longrightarrow f$ uniformly on compact subsets of $(0, \infty)$, then $\mathbf{Q}_{t} f_{n}(x) \longrightarrow \mathbf{Q}_{t} f(x)$ uniformly for $(t, x)$ in compact subsets of $(0, \infty) \times[0, \infty)$.

When $\sigma \geq 0$, nothing in Theorem 1 is surprising, with the possible exception of the regularity of solutions at the spacial boundary. Moreover, the solutions when $\sigma \geq 0$ admit a familiar probabilistic interpretation. Namely, when $\sigma=0$, the associated Markov process is simply Brownian motion with drift $\mu$ which is absorbed when it hits 0 . When $\sigma>0$, the associated Markov process is again Brownian motion with drift $\mu$, only now 0 is a "sticky" reflection point. More precisely, let $\left\{B_{t}: t \geq 0\right\}$ be a standard, $\mathbb{R}$-valued Brownian motion, and set

$L_{t}(x)=\max \left\{\left(x+B_{s}+\mu s\right)^{-}: s \in[0, t]\right\} \quad$ and $\quad X_{t}(x)=x+B_{t}+\mu t+L_{t}(x)$.

Then $\left\{X_{t}(x): t \geq 0\right\}$ is Brownian motion with drift $\mu$ reflected at 0 , and $\left\{L_{t}(x): t \geq 0\right\}$ is its local time at 0 . Finally, for any $\sigma \geq 0$, take

$$
\tau_{t}(x)=\inf \left\{\tau: \tau+\sigma^{-1} L_{\tau}(x) \geq t\right\}\left(\equiv \inf \left\{\tau: X_{\tau}(x)=0\right\} \text { when } \sigma=0\right) .
$$

Then $\left\{X_{\tau_{t}(x)}(x): t \geq 0\right\}$ is, depending on whether $\sigma=0$ or $\sigma>0$, Brownian motion with drift $\mu$ which is either absorbed at 0 or has a "sticky" reflection at 0 . In addition, an elementary application of Itô's calculus combined with Doob's stopping time theorem shows that

$$
u_{f}(t, x)=\mathrm{e}\left[f\left(X_{\tau_{t}(x)}(x)\right)\right] .
$$

For a complete account of one-dimensional diffusion equations which are amenable to probabilistic interpretation, see Dynkin's classic interpretation in [1] of Feller's theory.

\section{Preservation of Non-negativity when $\sigma<0$}

From (2) it is clear that $\left\{\mathbf{Q}_{t}: t \geq 0\right\}$ is a conservative, Markov semigroup when $\sigma \geq 0$, a conclusion which can be drawn (with much less effort) via a purely analytic minimum principle argument. On the other hand, when $\sigma<0$, the minimum principle is lost and $\left\{\mathbf{Q}_{t}: t \geq 0\right\}$ need not preserve non-negativity. In fact, we have (cf. Theorem 1.2 in [4]) the following. 
Theorem 2. Assume that $\sigma<0$. For $f \in F, u_{f}$ is non-negative if and only if $f$ is non-negative and

$$
f(0) \geq 2|\sigma| \int_{(0, \infty)} e^{2 \sigma \wedge \mu y} f(y) d y .
$$

Moreover, if $F(\sigma, \mu)$ is the subset of $f \in F$ which satisfy

$$
f(0)=2|\sigma| \int_{(0, \infty)} e^{2 \sigma \wedge \mu y} f(y) d y
$$

then $\left\{\mathbf{Q}_{t}: t \geq 0\right\}$ leaves $F(\sigma, \mu)$ invariant and its restriction to $F(\sigma, \mu)$ is Markov. Finally, $\mathbf{Q}_{t}$ frm $[o]--\leq$ frm $[o]--$ for all $t>0$, and equality holds if and only if $\sigma \geq \mu$.

Even without going into the details, it is reasonably easy to understand why the function $J(y) \equiv 2|\sigma| e^{2 \sigma \wedge \mu y}$ enters in the preceding. Namely, given a solution $u$, one can use integration by parts to see that

$$
\frac{d}{d t}(u(t, 0)-\langle J, u(t, \cdot)\rangle)=-2 \sigma(\mu-\sigma)^{+}(u(t, 0)-\langle J, u(t, \cdot)\rangle),
$$

where $\langle\varphi, \psi\rangle \equiv \int_{(0, \infty)} \varphi(y) \psi(y) d y$. Hence,

$$
u_{f}(t, 0)-\left\langle J, u_{f}(t, \cdot)=e^{-2 \sigma(\mu-\sigma)^{+} t}(f(0)-\langle J, f\rangle),\right.
$$

which makes it clear why $F(\sigma, \mu)$ is $\left\{\mathbf{Q}_{t}: t \geq 0\right\}$ invariant. More generally, (3) shows that

$$
f(0) \geq\langle J, f\rangle \Longrightarrow u_{f}(t, 0) \geq\left\langle J, u_{f}(t, \cdot)\right\rangle \quad \text { for all } t \geq 0 .
$$

Hence, if $f \geq 0$ and $f(0) \geq\langle J, f\rangle$, then an easy minimum principle argument shows that $u_{f} \geq 0$. Namely, choose $\eta \in C^{\infty}([0, \infty) ;(0,1))$ so that $\eta(0)>$ $\langle J, \eta\rangle, \frac{1}{2} \eta^{\prime \prime}+\mu \eta^{\prime}>-1$, and $\lim _{x \rightarrow \infty} \eta(x)=\infty$. For $\epsilon>0$, set $v_{\epsilon}(t, x)=$ $u_{f}(t, x)+\epsilon(t+\eta(x))$, and note that $\dot{v}_{\epsilon}>\frac{1}{2} v_{\epsilon}^{\prime \prime}+\mu v_{e}^{\prime}, v_{\epsilon}(t, 0)>\left\langle v_{\epsilon}(t, \cdot)\right\rangle$, $\lim _{t \rightarrow 0} v_{\epsilon}(t, \cdot)>0$, and, for each $t>0, \inf _{\tau \in(0, t]} v_{\epsilon}(\tau, x) \longrightarrow \infty$ as $x \rightarrow \infty$. From these it is easy to conclude that $v_{\epsilon} \geq 0$ everywhere for each $\epsilon>0$.

The proof that $u_{f} \geq 0 \Longrightarrow f(0) \geq\langle J, f\rangle$ is more challenging. One way to proceed is via probability theory. Namely, take (cf. (2)) $X_{t}=X_{t}(0)$ and $L_{t}=L_{t}(0)$, and define $\Psi_{t}=|\sigma|^{-1} L_{t}-t$ and $\zeta=\inf \left\{t: \Psi_{t}=0\right\}$. By Itô's calculus, one knows that $t \rightsquigarrow u_{f}\left(\Psi_{t}, X_{t}\right)$ is a local martingale on $[0, \zeta)$. Thus, if $u_{f} \geq 0$, then $t \rightsquigarrow u_{f}\left(\Psi_{t}, X_{t}\right)$ is a non-negative supermartingale on $[0, \zeta)$, and so

$$
\begin{aligned}
f(0) & =u_{f}(0,0) \geq \lim _{t \rightarrow \infty} \mathrm{e}\left[u_{f}\left(\Psi_{t \text { wedge } \zeta}, X_{t \wedge \zeta}\right)\right] \\
& =\mathrm{e}\left[f\left(X_{\zeta}\right), \zeta<\infty\right]+\lim _{t \rightarrow \infty} \mathrm{e}\left[u_{f}\left(\Psi_{t}, X_{t}\right), \zeta=\infty\right] .
\end{aligned}
$$


Similarly, if $u_{f}$ is bounded, then the inequality in the preceding can be replaced by equality. Hence

$$
\begin{aligned}
u_{f} \geq 0 & \Longrightarrow f(0) \geq \mathrm{e}\left[f\left(X_{\zeta}\right), \zeta<\infty\right] \\
u_{f} \text { bounded } & \Longrightarrow f(0)=\mathrm{e}\left[f\left(X_{\zeta}\right), \zeta<\infty\right]+\lim _{t \rightarrow \infty} \mathrm{e}\left[f\left(X_{t}\right), \zeta=\infty\right]
\end{aligned}
$$

At this point, one has to check that, with probability one,

$$
\begin{aligned}
& \mu>\sigma \Longrightarrow \lim _{t \rightarrow \infty} \Psi_{t}=-\infty \\
& \mu=\sigma \Longrightarrow \varlimsup_{t \rightarrow \infty} \Psi_{t}=\infty=-\varliminf_{t \rightarrow \infty} \Psi_{t} \\
& \mu<\sigma \Longrightarrow \lim _{t \rightarrow \infty} \Psi_{t}=\infty .
\end{aligned}
$$

In particular, if $\mu \geq \sigma$, then $\mathbb{P}(\zeta<\infty)=1$, and so the second line of (4) applied to $f \in F(\sigma, \mu)$ says that

$$
\langle J, f\rangle=\mathrm{e}\left[f\left(X_{\zeta}\right), \zeta<\infty\right] \quad \text { when } \mu \geq \sigma .
$$

That is, when $\mu \geq \sigma$, then $2|\sigma| e^{2 \sigma y}$ is the distribution of $X_{\zeta}$, and so the first line of (4) completes the proof that $u_{f} \geq 0 \Longrightarrow f(0) \geq\langle J, f\rangle$ when $\mu \geq \sigma$. The case when $\sigma<\mu$ is a little trickier. To handle it, one must first observe that, by the last line of (5), the second term on the right in the second line of (4) can be replaced by

$$
\mathrm{e}\left[\lim _{t \rightarrow \infty} u_{f}\left(t, X_{t}\right), \zeta=\infty\right]
$$

when $\mu<\sigma$. Secondly, one has to show that

$$
\mu<\sigma \& f \in F(\sigma, \mu) \Longrightarrow \lim _{t \rightarrow \infty} u_{f}(t, x)=0 \quad \text { for all } x \in[0, \infty) .
$$

Once this has been done, one can proceed as before to check that, when $\mu<\sigma, 2|\sigma| e^{2 \mu y}$ is the distribution of $X_{\zeta}$ on $\{\zeta<\infty\}$ and therefore $u_{f} \geq$ $0 \Longrightarrow f(0) \geq\langle J, f\rangle$.

\section{Bounded Solutions \& Long Time Behavior}

A closer examination of the probabilistic argument given in $\S 1$ reveals that, when $\sigma<0$,

$$
\begin{array}{r}
\mu \geq \sigma \Longrightarrow u_{f} \text { is bounded } \Longleftrightarrow f \in F(\sigma, \mu) \\
\mu<\sigma \Longrightarrow u_{f} \text { is bounded for all } f \in F \\
\text { and } \lim _{t \rightarrow \infty} u_{f}(t, \cdot)=0 \Longleftrightarrow f \in F(\sigma, \mu) .
\end{array}
$$

One gets more precise information from the following (cf. Theorem 3.2 in [3]). 
Theorem 3. Assume that $\sigma<0$. Given $f \in F$, set (cf. the notation introduced following Theorem 2) $\delta f=f(0)-\langle J, f\rangle$. Then, as $t \nearrow \infty$,

$$
\begin{aligned}
& \mu<\sigma \Longrightarrow u_{f}(t, x) \longrightarrow \frac{\mu}{\mu-\sigma} \delta f \\
& \mu=\sigma \Longrightarrow \frac{u_{f}(t, x)}{t} \longrightarrow 2 \sigma^{2} \delta f \\
& \mu>\sigma \Longrightarrow e^{2 \sigma(\mu-\sigma) t} u_{f}(t, x) \longrightarrow \frac{\mu-2 \sigma}{\mu-\sigma} e^{-2(\mu-\sigma) x} \delta f
\end{aligned}
$$

uniformly on compacts. In particular,

$$
\begin{aligned}
\delta f \geq 0 & \Longleftrightarrow \varlimsup_{t \rightarrow \infty} u_{f}(t, x) \geq 0 \text { for some } x \in[0, \infty) \\
& \Longleftrightarrow \varliminf_{t \rightarrow \infty} u_{f}(t, x) \geq 0 \text { for all } x \in[0, \infty) .
\end{aligned}
$$

The proof of Theorem 3 involves no probability theory. The idea is to write $u_{f}=(\delta f) v+u_{\tilde{f}}$, where $v$ is the solution with initial condition $\operatorname{frm}[o]--\{0\}$ and $\tilde{f}=f-(\delta f) f r m[o]--\{0\}$. Because $\tilde{f} \in F(\sigma, \mu)$, we know that $u_{\tilde{f}}$ is always bounded and, as $t \nearrow \infty$, tends to 0 if $\mu<\sigma$. Thus, everything comes down to the analysis of $v(t, \cdot)$ as $t \nearrow \infty$, and this analysis is carried out in Lemma 3.1 of [3].

\section{Bounded, Ancient Solutions}

Here we consider solutions to (1) which are ancient in the sense that they are solutions on $(-\infty, 0) \times[0, \infty)$, and our goal is to classify all ancient solutions which are bounded. Our first result about such a solution is contained in the following regularity result (cf. Lemma 4.2 in [3]).

Lemma 4. Assume that $u \in C^{1,2}((a, b) \times[0, \infty) ; \mathbb{R})$ is a bounded solution to (1) for some $-\infty<a<b<\infty$. Then, $u \in C^{\infty}((a, b) \times[0, \infty)$; $\mathbb{R})$ and there is $a K$, which depends only of $\sigma$ and $\mu$, such that, for each $n \geq 1$ and $t \in(a, b)$,

$$
\left\|\partial_{x}^{n} u(t, \cdot)\right\|_{\mathrm{u}} \leq\left(\frac{K n}{t-a}\right)^{\frac{n}{2}} e^{K(t-a)}\|u\|_{\mathrm{u}} .
$$

In particular, if $u$ is a bounded, ancient solution, then $u \in C_{\mathrm{b}}^{\infty}((-\infty, 0) \times$ $[0, \infty) ; \mathbb{R})$ and there exists a $K^{\prime} \in[1, \infty)$, depending only on $\sigma$ and $\mu$, such that

$$
\|u\|_{C_{\mathrm{b}}^{n, 2 n}((-\infty, 0) \times[0, \infty) ; \mathbb{R})} \leq\left(K^{\prime}\right)^{n}\|u\|_{\mathrm{u}}
$$

for all $n \geq 0$. Hence, each bounded ancient solution admits a unique continuation as an entire, holomorphic function on $\mathbb{C}^{2}$. 
Theorem 5. If $u$ is a bounded, ancient solution to (1), then

$$
u(t, x)=A+B e^{-2(\mu-\sigma)^{+}(\sigma t+x)}
$$

for some $(A, B) \in \mathbb{R}^{2}$, where $B=0$ if either $\sigma \geq \mu$ or $0<\sigma<\mu$. Moreover, if $u$ is a bounded solution to (1) in the whole of $\mathbb{R} \times[0, \infty)$, then $u$ is constant.

Given the preceding lemma, the final assertion is an easy corollary of the initial statement. In order to prove the initial statement, one considers the function $w=\sigma u^{\prime}-u$, which, by the lemma, is a bounded solution to $\dot{w}=\frac{1}{2} w^{\prime \prime}+\mu w^{\prime}$ in $(-\infty, 0) \times[0, \infty)$ which vanishes on the spacial boundary $(-\infty, 0) \times\{0\}$. The desired result will follow once we show that such a $w$ is constant in $t \in(0, \infty)$. To this end, let $Q(t, x, y)$ be the heat kernel for $\frac{1}{2} \partial^{2}+\mu \partial$ in $(0, \infty)$ with boundary condition 0 at 0 . Then

$$
w\left(t_{2}, x\right)-w\left(t_{1}, x\right)=\int_{(0, \infty)} Q(T, x, y)\left(w\left(t_{2}-T, y\right)-w\left(t_{1}-T, y\right)\right) d y .
$$

Since

$$
\lim _{T \rightarrow \infty} \int_{(0, \infty)} Q(T, x, y) e^{-2 \mu^{+} y} d y=0,
$$

it suffices to show that

$$
\left|w\left(t_{2}, y\right)-w\left(t_{1}, y\right)\right| \leq 2\|w\|_{\mathrm{u}} e^{-2 \mu^{+} y} \quad \text { for all } t_{1}<t_{2}<0 \text { and } y \geq 0 .
$$

A proof of $\left(^{*}\right)$ can be based on a coupling argument. Namely, set $h=t_{2}-t_{1}$, and let $\left\{B_{t}: t \geq 0\right\}$ and $\left\{B_{t}^{\prime}: t \geq 0\right\}$ be a pair of mutually independent, $\mathbb{R}$-valued Brownian motions starting at 0 . Next, define $\tau$ to be the first time at which the path $t \rightsquigarrow y+B_{t+h}+\mu(t+h)$ crosses the path $t \rightsquigarrow y+B_{t}^{\prime}+\mu t$. Equivalently, $\tau=\inf \left\{t \geq 0: B_{t}^{\prime}-B_{t+h}=\mu h\right\}$. Then $\mathbb{P}(\tau<\infty)=1$, and

$$
t \rightsquigarrow U_{t} \equiv B_{t \wedge \tau}^{\prime}+\left(B_{t+h}-B_{t \wedge \tau+h}\right)
$$

is again a Brownian motion starting at 0 . Because $y+B_{t+h}+\mu(t+h)=$ $y+U_{t}+\mu t$ when $\tau \leq t,\left|w\left(t_{2}, y\right)-w\left(t_{1}, y\right)\right|$ is equal to

$$
\begin{aligned}
& \lim _{t \rightarrow \infty} \mid \mathrm{e}\left[w\left(t_{1}-t, y+B_{t+h}+\mu(t+h)\right), \zeta_{y}^{B}>t+h\right] \\
& -\mathrm{e}\left[w\left(t_{1}-t, y+U_{t}+\mu t\right), \zeta_{y}^{U}>t\right] \mid \\
& =\lim _{t \rightarrow \infty} \mid \mathrm{e}\left[w\left(t_{1}-t, y+U_{t}+\mu t\right), \zeta_{y}^{B}>t+h\right] \\
& -\mathrm{e}\left[w\left(t_{1}-t, y+U_{t}+\mu t\right), \zeta_{y}^{U}>t\right] \mid \\
& \leq\|w\|_{\mathrm{u}} \lim _{t \rightarrow \infty}\left(\mathbb{P}\left(\zeta_{y}^{B}>t+h \& \zeta_{y}^{U} \leq t\right)+\mathbb{P}\left(\zeta_{y}^{B} \leq t+h \& \zeta_{y}^{U}>t\right)\right) \\
& \leq\|w\|_{\mathrm{u}}\left(\mathbb{P}\left(\zeta_{y}^{U}<\infty\right)+\mathbb{P}\left(\zeta_{y}^{B}<\infty\right)\right)=2\|w\|_{\mathrm{u}} \mathbb{P}\left(\zeta_{y}^{B}<\infty\right),
\end{aligned}
$$

where $\zeta_{y}^{B}$ and $\zeta_{y}^{U}$ are, respectively, the first time that $t \rightsquigarrow y+B_{t}+\mu t$ and $t \rightsquigarrow y+U_{t}+\mu t$ hit 0 . Because $\mathbb{P}\left(\zeta_{y}^{B}<\infty\right)=e^{-2 \mu^{+} y},(*)$ follows. 


\section{A Harnack Principle}

At first sight, (1) appears to be a parabolic equation. As such, one should expect that, if it satisfies a Harnack principle at all, that principle will be "one-sided." For example, when $\sigma=0$,

$$
w_{\lambda}(t, x)=e^{\lambda x+\frac{\mu^{2}+\lambda^{2}}{2} t} \sinh (\lambda x)
$$

is a non-negative solution in $\mathbb{R} \times[0, \infty)$ for each $\lambda>0$, and although

$$
\sup _{\lambda>0} \frac{w_{\lambda}(s, x)}{w_{\lambda}(t, y)}
$$

is bounded for each $0<s<t$ and all $(x, y)$ in compact subsets of $(0, \infty)$, it is infinite whenever $s>t$ or $s=t$ but $x \neq y$; and the same sort of one-sided Harnack principle holds whenever $\sigma \geq 0$. Thus, it is somewhat intriguing that a more robust Harnack principle holds as soon as $\sigma<0$. To wit, one can prove (cf. the subsection following Theorem 5.6 in [3]) the following statement.

Theorem 6. Assume that $\sigma<0$. For each $0<\ell<L$ and $0<r<R$, there exists a $K<\infty$, depending only on $\sigma, \mu, \ell, L, r$, and $R$, such that $u(s, x) \leq$ $K u(t, y)$ whenever $(s, t) \in[0, \ell]^{2},(x, y) \in[0, r]^{2}$, and $u$ is a non-negative solution to $(1)$ in $(-L, L) \times[0, R)$. Moreover, if $\mathcal{U}(L, R)$ is the set of all nonnegative solutions to $(1)$ in $(-L, L) \times[0, R)$, then, for each $(s, x) \in(-L, L) \times$ $[0, R),\{u \in \mathcal{U}(L, R): u(s, x) \leq 1\}$ is compact in $C^{\infty}((-L, L) \times[0, R) ;[0,1])$.

In broad outline, the proof of this Harnack principle follows a line of reasoning which is familiar to experts in such matters. Namely, one shows that, for $(s, x) \in[-\ell, \ell] \times[0, r], u(s, x)$ is bounded above and below by positive multiples of $u\left(\ell^{\prime}, 0\right)$ plus the integral of $u$ over $\left[-\ell^{\prime}, \ell^{\prime}\right] \times\left[0, r^{\prime}\right]$, where $\ell<\ell^{\prime}<L$ and $r<r^{\prime}<R$. The most interesting part comes when one checks the lower bound, which comes from thinking about the behavior of $\Psi_{t}$. Namely, although $\Psi_{t}$ does nothing but decrease while $X_{t}>0$, it increases very rapidly whenever $X_{t}$ visits 0 , and it this increase which accounts for the "backdoor elliptic" nature of the resulting Harnack principle. In this connection, it should be observed that one cannot localize Harnack principle here to regions which do not include a healthy component of the spacial boundary.

\section{Non-Negative, Ancient Solutions}

When $\mu \neq \sigma$ and $\sigma \neq 0$,

$$
\left.e^{-\mu x+\frac{\lambda^{2}-\mu^{2}}{2} t}(\cosh (\lambda x))+\frac{\lambda^{2}+\sigma^{2}-(\mu-\sigma)^{2}}{2 \lambda \sigma} \sinh (\lambda x)\right)
$$


is a non-constant, non-negative global (i.e., on $\mathbb{R} \times[0, \infty)$ ) solution to (1) for every $\lambda>0$ with the property that $\frac{\lambda^{2}+\mu^{2}-(\mu-\sigma)^{2}}{2 \lambda \sigma}>-1$. When $\sigma=0$,

$$
e^{-\mu x+\frac{\lambda^{2}-\mu^{2}}{2} t} \sinh (\lambda x)
$$

is a non-constant, non-negative, global solution to (1) for each $\lambda>0$. Finally, in the balanced case (i.e., $\sigma=\mu$ ), if $\sigma \geq 0$, then

$$
e^{-\mu x+\frac{\lambda^{2}-\mu^{2}}{2} t}\left(\sinh (\lambda x)+\frac{2 \lambda \mu}{\lambda^{2}+\mu^{2}} \cosh (\lambda x)\right)
$$

is a non-constant, non-negative, global to (1) for each $\lambda>0$. On the other hand, in the balanced case, if $\sigma<0$, all non-negative, global solutions to (1) are constant. An understanding of this last result can be found in (5). Namely, the middle line of (5) makes it reasonably easy to show that the process $\left\{\left(\Psi_{t}, X_{t}\right): t \geq 0\right\}$ is recurrent. Thus, since each non-negative, global solution is a non-negative supermartingale along this process, the constancy of such solutions follows from a standard argument based on Doob's martingale convergence theorem.

Of course, the situation is completely different when one considers nonnegative ancient solutions. Indeed, even in the balanced case with $\sigma<0$, there are lots of non-constant, non-negative ancient solutions. The reason why such ancient solutions can exist even though global ones cannot is that the recurrence, alluded to above, disappears if the process is stopped when $\Psi_{t}$ first visits 0 , and it is the stopped process along which non-negative, ancient solutions will be non-negative supermartingales. In an attempt to understand what is the structure of the set of non-negative, ancient solutions in the balanced case, we proved the following.

Theorem 7. When $\sigma=-1=\mu$, there is a one-to-one correspondence between non-negative solutions $u$ to (1) and triples $(a, b, \nu),(a, b) \in[0, \infty)^{2}$ and $\nu$ a measure on $[0, \infty)$ with $\int e^{\lambda c} \nu(d c)<\infty$ for all $\lambda>0$, given by

$$
u(\psi, x)=a+b(x-\psi)+\int_{0}^{\infty} h_{1+c}(\psi, x) \nu(d c)
$$

where

$$
\begin{aligned}
h_{1+c}(\psi, x) \equiv & 4(c+1)\left[1-e^{\left(\frac{1}{2} c^{2}+c\right) \psi-c x}\right] \\
& +c^{2} e^{\left(\frac{1}{2} c^{2}+c\right) \psi}\left[e^{(2+c) x}-e^{-c x}\right] .
\end{aligned}
$$

Given the requisite computations of the Green's function involved, our proof of this result is a straight-forward application of Martin's boundary theory. In order to interpret its conclusion as a statement about the associated Martin compactification, let $Q$ denote the second quadrant $(-\infty, 0) \times[0, \infty)$ in the Euclidean plane. Next, compactify $Q$ by adjoining the line $\{0\} \times[0, \infty)$ and 
the quarter circle $\left[\frac{1}{2} \pi, \pi\right]$ at infinity. Now identify all points on $\{0\} \times[0, \infty)$ with a single point $\alpha$ and all points at infinity having "angle" in $\left[\frac{3}{4} \pi, \pi\right]$ with a (different) single point $\gamma$. That is, if $\left\{\left(\psi_{n}, x_{n}\right)\right\}_{1}^{\infty} \subseteq Q$ converges to a point on $\{0\} \times[0, \infty)$, we say that $\left(\psi_{n}, x_{n}\right) \longrightarrow \alpha$; and if it converges to infinity in such a way that $\arctan \frac{\psi_{n}}{x_{n}}$ converges to a point in $\left[\frac{3}{4} \pi, \pi\right]$, we say that $\left(\psi_{n}, x_{n}\right)$ tends to $\gamma$. Finally, if $\left\{\left(\psi_{n}, x_{n}\right)\right\}_{1}^{\infty}$ tends to infinity so that $\arctan \frac{\psi_{n}}{x_{n}}$ converges to $\beta \in\left[\frac{1}{2} \pi, \frac{3}{4} \pi\right]$, we will say that $\left(\psi_{n}, x_{n}\right) \longrightarrow \beta$. With these conventions,

$$
\{\alpha\} \cup\left[\frac{1}{2} \pi, \frac{3}{4} \pi\right] \cup\{\gamma\}
$$

can be identified with the Martin boundary of $Q$ in such a way that the associated Martin kernel with reference point $\left(\psi_{0}, x_{0}\right) \in Q$ is given on the boundary by

$$
\begin{gathered}
\kappa((\psi, x) ; \alpha)=\frac{x-\psi}{x_{0}-\psi_{0}}, \quad \kappa((\psi, x) ; \gamma)=1, \\
\kappa((\psi, x) ; \beta)=\frac{h_{-\tan \beta}(\psi, x)}{h_{-\tan \beta}\left(\psi_{0}, x_{0}\right)} .
\end{gathered}
$$

\section{References}

1. E.B. Dynkin. Markov Processes. Number 122 in Grundlehren Series. SpringerVerlag, 1965.

2. W. Feller. Diffusion processes in one dimension. TAMS, pages 1-31, 1954.

3. D. Stroock and D Williams. Further study of a simple pde. To appear in the volume of the Illinois J. of Math. dedicated to the memory of J.L. Doob.

4. D. Stroock and D. Williams. A simple pde and wiener-hopf riccati equations. Comm. Pure \& Appl. Math., 58(8):1116-1148, 2005. 